\title{
Anuncios entre vídeos, juegos y fotos. Impacto publicitario que recibe el menor a través del teléfono móvil
}

\section{Ads in videos, games, and photos: Impact of advertising received by children through mobile phones}

\author{
Beatriz Feijoo-Fernández; Charo Sádaba-Chalezquer; Simón Bugueño-Ipinza
}

Cómo citar este artículo:

Feijoo-Fernández, Beatriz; Sádaba-Chalezquer, Charo; Bugueño-Ipinza, Simón (2020). "Anuncios entre vídeos, juegos y fotos. Impacto publicitario que recibe el menor a través del teléfono móvil". Profesional de la información, v. 29, n. 6, e290630.

https://doi.org/10.3145/epi.2020.nov.30

Artículo recibido el 27-04-2020

Aceptación definitiva: 05-06-2020

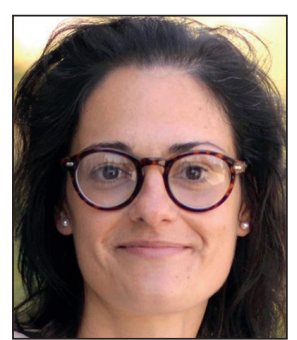

Beatriz Feijoo-Fernández $\square$ https://orcid.org/0000-0001-5287-3813

Universidad Internacional de la Rioja Facultad de Empresa y Comunicación Avenida de La Paz, 137 26006 Logroño, España beatriz.feijoo@unir.net

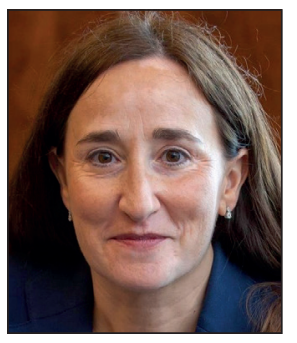

Charo Sádaba-Chalezquer https://orcid.org/0000-0003-2596-2794

Universidad de Navarra Facultad de Comunicación Campus universitario 31009 Pamplona, España csadaba@unav.es

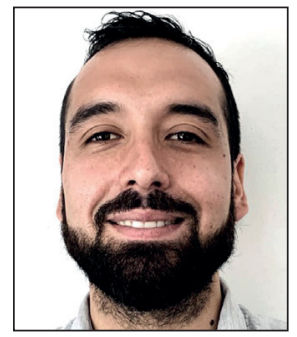

Simón Bugueño-Ipinza

https://orcid.org/0000-0003-4483-1458

Visiona RSE y Gestión Social Limitada

Las Nieves, 3331, Vitacura

Santiago, Chile

sbuguenno@gmail.com

\section{Resumen}

Estudio exploratorio sobre la densidad publicitaria a la que se enfrentan los menores cuando emplean el teléfono móvil. Pasan gran parte de su tiempo conectados en plataformas como YouTube, apps de juegos e Instagram, en las que existe un nivel de exposición publicitaria (14 minutos por hora de uso, o 23\%) ligeramente superior al de medios como la televisión, llegando en algunos momentos a estar presente la publicidad más del $80 \%$ del tiempo de navegación. Esto muestra el estudio realizado en el Área Metropolitana de Santiago de Chile con niños de 10 a 14 años con el objetivo de registrar y analizar su exposición a la publicidad recibida a través de un teléfono móvil con conexión a internet. El análisis también recoge que la interacción de los niños con el contenido publicitario al que acceden es sobre todo visual (contacto sólo ocular) y el mayor porcentaje de anuncios que reciben es en los juegos online (23\%), seguidos de la restauración y distribución (18\%), entretenimiento (8\%) y moda (8\%). Estos datos se han obtenido mediante una observación digital no participante en la que se propuso al usuario que hiciera una grabación de vídeo de la pantalla del teléfono con el que accedía frecuentemente (screen recorder) y así quedasen registrados los contenidos que visitase. Se accedió a una muestra de 45 menores que se sometieron a un seguimiento semanal, consiguiendo finalmente un cómputo de registros

Financiación

Este trabajo de investigación se circunscribe al proyecto Fondecyt Iniciación n. 11170336 con el título "El menor de edad como consumidor. Análisis de la publicidad que recibe el usuario de 10 a 14 años a través de los dispositivos móviles", financiado por la Comisión Nacional de Investigación Científica y Tecnológica (Conicyt) del Gobierno de Chile. 
audiovisuales superior a los 300, cada uno con una duración mínima que garantizase la viabilidad de la observación. Finalmente, el material recabado se sometió a un análisis de contenido.

\title{
Palabras clave
}

Menores de edad; Niños; Publicidad; Teléfonos móviles; Aplicaciones; Impacto publicitario; Saturación publicitaria; Etnografía digital; Juegos; Medios sociales; YouTube; Instagram.

\begin{abstract}
This study adopted an exploratory approach to try to establish lines of investigation on the advertising density that children face when using their mobile phones. While connected, minors are exposed to a slightly higher volume of advertising (14 minutes per hour of use) than via other media such as television, with presence of ads during more than $80 \%$ of browsing time. Data on exposure of children to advertising through mobile phones with an internet connection were collected and analyzed. The minors were aged 10 to 14 years and lived in Santiago, a metropolitan area in Chile. Children visually interact with and consume advertising content through mobile devices. This eye contact and consumption occur mainly when minors visit game applications, YouTube (app), and Instagram (app). Commercial content appeared $23.4 \%$ of the time analyzed. Online gaming (23\%), food and distribution (18\%), entertainment (8\%), and fashion (8\%) represented the highest percentage of ads. A non-participatory digital observation methodology was applied for data acquisition. Minors were asked to video-record the screen during common activities, thus registering their movements and the content to which they were visually exposed. Forty-five (45) children underwent weekly follow-ups, and an audiovisual database of over 300 recordings was created. Recordings meeting methodological constraints (on, for instance, duration) were subjected to content analysis.
\end{abstract}

\section{Keywords}

Children; Minors; Advertising; Ads; Adverts; Mobile phones; Smartphones; Apps; Impact of advertising; Advertising saturation; Digital ethnography; Games; Social media; YouTube; Instagram.

\section{Introducción}

El interés de la investigación por la relación entre los niños y la publicidad no es nuevo y tiene múltiples vertientes. De todas ellas da buena cuenta la revisión realizada por De-Jans et al. (2017) quienes repasaron los trabajos sobre niños y publicidad publicados entre 2006 y 2016. De acuerdo con su análisis hay cinco áreas que agrupan la mayoría de las investigaciones realizadas en este período:

- efectos de la publicidad;

- procesamiento de mensajes publicitarios;

- contenido y características de la publicidad dirigida a niños;

- influencias sociales;

- softwares, acciones y estrategias para proteger y empoderar a los niños ante los contenidos comerciales.

Su trabajo también señalaba que la relación entre los menores y la publicidad en el entorno digital está todavía pendiente de más investigación (Livingstone, 2003; Moore, 2004) y de nuevos enfoques metodológicos que se adapten mejor al tipo y modo de interacción de niños y adolescentes con los contenidos digitales.

Son varios los estudios sobre publicidad y niños en el entorno digital, sobre todo:

- su exposición a publicidad de alimentos, alcohol y tabaco (Culp; Bell; Cassady, 2010; Harris et al., 2012);

- presencia de publicidad en juegos, videojuegos u otras opciones de entretenimiento dirigidas a niños (An; Jin; Park, 2014; Waiguny; Nelson; Terlutter, 2012).

Sin embargo, el análisis de la publicidad que se consume a través de los móviles está todavía por estudiar pese a su evidente interés. El smartphone es la pantalla más importante de acceso a internet de este grupo de edad (Sádaba-Chalezquer, 2018; Mascheroni; Ólafsson, 2014). Además, algunas de sus características, como su uso personal, su ubicuidad, su movilidad o su tamaño incrementan la sensación de intimidad y por tanto, puede producir una relación con el contenido que se consume a través de él significativamente distinta, generando una transferencia emocional positiva de la pantalla (Vanwesenbeck; Walrave; Ponnet, 2017).

Como se ha mencionado, en el caso de los más pequeños la pantalla móvil se ha convertido en una de sus principales vías de acceso a internet. No en vano es llamativo que la mayor parte de los padres y madres cuando son preguntados por los mensajes comerciales a los que se enfrentan sus hijos señalen a la televisión como principal fuente de publicidad, mientras que estudios en otros países (Ofcom, 2016) ya indican que el consumo digital supera con creces al de la televisión convencional entre este grupo de edad. El estudio de Meyer et al. (2019), por ejemplo, analizó 39 apps utilizadas por niños entre 1 y 5 años y en el $95 \%$ de ellas encontró al menos un tipo de mensaje publicitario.

El análisis de la publicidad que se consume a través de los móviles está todavía por estudiar pese a su evidente interés 


\section{Relación del menor con la publicidad digital}

Las nuevas generaciones han crecido en un contexto mediático en el que consumen contenidos bajo demanda, lo que provoca que sus expectativas y exigencias con los mensajes publicitarios sean altas. Así lo demuestra el estudio AdReaction de Kantar Millward Brown (2017) que, tras encuestar a más de 23.000 consumidores de 40 países revela que las audiencias más jóvenes consideran molesta la publicidad digital, una actitud que se suaviza si sienten que tienen la posibilidad de controlar el contenido, saltándolo o cerrándolo. Por ello les molestan especialmente los formatos publicitarios invasivos como los pre-rolls no evitables o los anuncios en ventanas emergentes.

No obstante, estas nuevas audiencias, en comparación con anteriores generaciones, no rechazan la publicidad ni las marcas sino que eligen el contenido publicitario por el que sienten verdadero interés. De ahí que tengan una actitud más positiva ante comerciales con recompensas, que usen efectos especiales, nuevos elementos de inmersión o que muestren a celebridades famosas. Los anuncios que no recurren a estas técnicas presentan una baja efectividad, como los publicados en apps de videojuegos (recurrentes en esta investigación), pero a los que rara vez se le presta atención, al contrario de lo que sucede con los integrados en Instagram por ejemplo, que ofrecen un nivel de recuerdo mayor. Así lo confirma un estudio in-

Las nuevas audiencias, en comparación con anteriores generaciones, no rechazan la publicidad ni las marcas sino que eligen el contenido publicitario por el que sienten verdadero interés dependiente realizado por las empresas Kargo Research

y Eye Tracking Tobii Pro (2019). En esencia, el público más joven es quien demanda formatos que presentan límites indefinidos entre entretenimiento, información y publicidad, en los que resulta más difícil para la audiencia reconocer su intencionalidad persuasiva (Oates; Newman; Tziortzi, 2014).

Una encuesta realizada por YouGov (2017) sobre publicidad y adolescentes en Estados Unidos concluyó que este público está dividido a la hora de confiar en los anuncios que ven, leen o escuchan en el mundo digital. En los dispositivos móviles, los canales que generan más confianza para recibir publicidad coinciden con las apps que más descargan, principalmente YouTube, WhatsApp y juegos, lo que lleva a deducir que tienden a fiarse más de los inputs publicitarios que reciben a través de plataformas en las que mejor se manejan (De-Frutos-Torres; Pretel-Jiménez; Sánchez-Valle, 2014; Feijoo-Fernández; García-González, 2019). Es en estas plataformas, especialmente en los juegos, donde diversos autores han evidenciado cierta "anarquía" con respecto a la diferenciación y categorización de los mensajes publicitarios (An; Kang, 2014; Chen et al., 2013; Terlutter; Capella, 2013).

Ante este panorama, las implicaciones de profundizar en la relación entre publicidad, niños y dispositivos móviles son numerosas y de gran calado. Y quizá las más significativas tienen que ver con el enriquecimiento y actualización de los programas y prácticas de educomunicación o alfabetización mediática. Si la interacción con contenidos de entretenimiento, de información o de persuasión tiene lugar cada vez con más frecuencia en entornos digitales mediados por el móvil, es razonable actualizar los programas dirigidos a la adquisición y desarrollo de conocimiento y capacidades críticas y de seguridad que tengan en cuenta estos parámetros. No sólo los móviles facilitan nuevos mensajes y narrativas que se adaptan a pantallas más pequeñas y personales, sino que también suscitan unas emociones que pueden condicionar el espíritu crítico (Rozendaal et al., 2011; Oates; Newman; Tziortzi, 2014). En el caso de la alfabetización mediática muchas voces postulan que hay que primar el enfoque actitudinal ya que el meramente conceptual se muestra limitado (Koslow, 2000; Rozendaal et al., 2013; An; Jin; Park, 2014; Vanwesenbeeck; Walrave; Ponnet, 2017; Van-Reijmersdal et al., 2017; Hudders et al., 2017). Saber identificar un contenido como publicidad no es suficiente para activar el espíritu crítico y el escepticismo sobre su evidente fin persuasivo. En línea con lo reclamado por la OCDE (2011), las evidencias científicas deberían ser la base sobre la que construir políticas públicas de actuación también en lo que se refiere a las pantallas y los menores.

Los resultados de este estudio podrían también ayudar a padres y madres, en la mayor parte de los casos los artífices y facilitadores de que los más pequeños tengan acceso móvil a internet, a establecer pautas que aseguren un uso más seguro que minimice los riesgos de su contacto con contenidos publicitarios.

\section{La conexión móvil en el contexto chileno}

Chile presenta un interesante caso de estudio en relación con el acceso y consumo de internet. Según la IX Encuesta de acceso y usos de internet promovida por la Subsecretaría de Telecomunicaciones de Chile (Subtel, 2017), el 87,4\% de los hogares chilenos dispone de acceso propio y pagado a internet, siendo la conexión móvil el principal modo de acceso dentro del hogar (29,6\%), tras la conexión fija (28,9\%). Los domicilios que disponen de ambas conexiones representan el 27,2\%. El Estudio de Móviles en Latinoamérica desarrollado por IMS (IMS; Comscore, 2017) pone de manifiesto que Chile es el país de la región con un mayor nivel penetración del servicio de internet entre sus habitantes, seguido de Argentina, México y Brasil.

En la línea de lo anterior, el 84,6\% de los accesos a internet se produce a través de un dispositivo móvil, preferentemente desde un smartphone, que representa casi el $80 \%$ del total de esos accesos (Subtel, 2019). Es más, la población chilena dispone de un equipamiento de teléfonos inteligentes al nivel de países como Alemania, Canadá o Reino Unido (Pew 
Research Center, 2018), a través del que se realiza un consumo mensual de internet promedio por usuario de 10 gigabytes, cifra similar a la que se observa en regiones de mayor desarrollo económico (Subtel, 2019). De estos datos se desprende que la principal puerta de acceso a la Red entre la población chilena son los servicios móviles, tanto dentro como fuera del hogar.
Los resultados de este estudio pueden ayudar a padres y madres a establecer pautas que aseguren un uso más seguro que minimice los riesgos del contacto de los menores con contenidos publicitarios

Esta penetración de la tecnología móvil no es ajena a los niños chilenos. Según datos del último estudio sobre usos, oportunidades y riesgos en el uso de las TIC en Chile del proyecto Kids Online (Cabello; Claro, 2017), un 92\% de niños y adolescentes usuarios de internet dispone de smartphone y cada vez a edades más tempranas: según eMarketer (2016), un $60 \%$ de los adolescentes latinoamericanos recibe su primer teléfono móvil a los 12 años. Aunque se dan diferencias significativas en el equipamiento tecnológico en función del estrato socioeconómico (C1-C2, C3, D-E) y del hábitat (urbano o rural) (Cabello et al., 2018), la penetración del teléfono inteligente es la más uniforme socialmente en comparación con otras pantallas como el ordenador de escritorio o portátil.

El presente artículo busca aportar más evidencias sobre cómo es la relación entre los menores chilenos y la publicidad que consumen a través de sus dispositivos móviles, empleando para ello un enfoque metodológico innovador que primó el registro real de sus rutinas de navegación en los propios aparatos de uso diario.

\section{Metodología}

Esta investigación tiene lugar en el contexto de un proyecto de mayor envergadura titulado "El menor de edad como consumidor. Análisis de la publicidad que recibe el usuario de 10 a 14 años a través de los dispositivos móviles", en cuya primera fase se abordó el nivel de detección, actitud y confianza que los menores (10-14 años) manifiestan ante la publicidad que reciben en sus dispositivos móviles, mediante un cuestionario suministrado en 501 hogares del Área Metropolitana de Chile. Se definió este grupo de edad porque se confirma (Cabello; Claro, 2017) que en la franja de los 10-12 años se incrementa grandemente la posesión personal del teléfono móvil.

Enmarcado en la segunda fase del proyecto, este artículo busca conocer el impacto publicitario real que los menores reciben a través de sus teléfonos móviles en su rutina diaria.

Para responder al objetivo planteado se formularon las siguientes preguntas de investigación:

1. ¿Qué porcentaje de tiempo el menor está expuesto a publicidad mientras utiliza su teléfono móvil?

2. ¿Sobre qué categorías de productos y marcas están recibiendo publicidad los menores de edad a través del teléfono móvil?

3. ¿Qué tipo de relación mantiene el menor con los mensajes publicitarios que recibe en el dispositivo?

4. ¿Marca la propiedad del teléfono diferencias en la exposición publicitaria del menor ante este tipo de pantalla?

5. ¿A través de qué aplicaciones reciben más publicidad los menores?

Para indagar en la relación de los menores con la publicidad, se recurrió al uso de técnicas que permitiesen profundizar de una manera directa en la exposición publicitaria del menor ante la pantalla del móvil. Para ello se ha empleado el método de la etnografía digital con el objetivo prioritario de conseguir información lo más fiel posible a la realidad que se quería estudiar, en este caso, el impacto publicitario que el niño recibe mientras emplea el móvil.

La etnografía digital consiste en una adaptación al estudio de las comunidades online (Hine, 2004), método más rápido, más sencillo y menos costoso que la etnografía tradicional, y más naturalista y discreto que los grupos de discusión o entrevistas, permitiendo la relación de información pública no inductiva sobre el simbolismo, los significados y los patrones de consumo de grupos determinados online sin preguntarles, sino desde el análisis de su rutina espontánea con el dispositivo. Esta técnica ha sido extensamente utilizada por investigadores en temas relacionados con la inclusión digital y la apropiación de internet en un entorno "natural" (Bakardjieva, 2005; Leung, 2005; Miller; Slater, 2000). Entre sus ventajas se encuentran que permite:

- observar directamente cómo se desenvuelven los sujetos, sus actitudes y creencias;

- capturar aspectos que son difíciles de poner en palabras;

- acceso privilegiado a información que de otra forma puede ser difícil de obtener.

La metodología utilizada en este estudio se basó en una observación digital no participante por parte del equipo de investigación para analizar qué mensajes publicitarios recibe el niño a través del teléfono móvil al que tenía acceso. Para ello, el propio menor realizó una grabación de vídeo de la pantalla de su dispositivo (screen recorder) con la que se registraron todos sus movimientos y contenidos visitados en su uso del aparato móvil.

La naturaleza de este proyecto invita a una serie de reflexiones sobre la aplicación del método etnográfico y con las consideraciones éticas a tener en cuenta, particularmente por la participación de menores de edad en el trabajo de campo. Con el objetivo de resguardar la integridad de los participantes del estudio y de los investigadores, para la recolección de la información se solicitó autorización al tutor mediante la firma de un consentimiento informado y también al propio 
menor a través de un asentimiento, documentos previamente revisados y validados por el Comité de Ética de la universidad al que está adscrito el proyecto de investigación (Universidad de Los Andes, Chile).

Finalmente, el propio participante o el tutor realizó el envío de las piezas audiovisuales, de una duración mínima que garantizase la calidad de la observación, vía WhatsApp o email a los responsables de la investigación. El seguimiento a los menores se produjo durante una semana (de lunes a domingo) generando idealmente una grabación por día. En las instrucciones facilitadas al adulto responsable se recomendaba que se procediese a la toma de datos en el contexto rutinario en el que el menor usa el dispositivo, con el objetivo de que la recogida de información fuese lo más fidedigna posible a la realidad del usuario. El carácter no participante del método conllevó que las grabaciones se realizasen sin la supervisión del investigador, lo que puede suponer una limitación a la investigación. Este trabajo de campo se realizó entre mayo y septiembre de 2019.

Para el análisis del material audiovisual se estableció como variable de análisis fundamental, además del sexo y de la edad, la propiedad del teléfono móvil en el que operaba el niño, categorizando la muestra en función de si poseía en exclusiva un aparato o accedía a uno propiedad de sus padres. Esta distinción resultó fundamental para contextualizar el impacto publicitario a través de este dispositivo que se rige por el historial de navegación de la web. De ahí la importancia de que se realizase la observación desde los propios móviles del hogar, no mediante dispositivos adquiridos expresamente para la investigación.

El resultado de la fase de campo se tradujo en el registro de los teléfonos móviles de 45 menores (móvil padre N=17, móvil propio $\mathrm{N}=28$; sexo masculino $\mathrm{N}=14$, sexo femenino $\mathrm{N}=31$; Edad 10-12 años $\mathrm{N}=21$, Edad 13-14 años $\mathrm{N}=24$ ), que se sometieron a la monitorización. Estos menores ya habían participado en la primera fase cuantitativa del proyecto de investigación y se seleccionaron por presentar un perfil de uso del teléfono móvil relevante para el estudio y de acuerdo a las variables de análisis definidas para esta etapa (edad, sexo y tipo de propiedad del dispositivo). Hay que tener en cuenta que la participación de carácter voluntario, la necesidad de mantener un compromiso y constancia durante el trabajo de campo, además de lo invasivo del método, dificultó la definición final de la muestra. De todos modos, se logró la recolección de más de 300 grabaciones que supusieron un total de 41 horas, 45 minutos y 39 segundos de grabación.

Si bien para el levantamiento de la información se siguió el método de la etnografía digital, para el tratamiento del registro audiovisual obtenido se realizó un análisis de contenido para conocer desde un enfoque descriptivo y exploratorio la cantidad de publicidad que reciben los menores en su uso habitual del móvil. El análisis de contenido se materializó en una matriz compuesta por las siguientes variables de clasificación:

1. Edad.

2. Sexo.

3. Propiedad del teléfono móvil.

4. Sitio web o aplicación en la que se navega.

5. Identificación de si en el sitio web o aplicación se expone a publicidad o no.

6. Tiempo de exposición a cada publicidad.

7. Tipo de relación con la publicidad:

- visual: aquella donde el menor sólo visualiza la publicidad;

- interactúa: el usuario presta atención publicitaria y genera algún tipo de interacción con ella como cerrar u observarla por un tiempo prolongado;

- click: hace click sobre ella y redirecciona la navegación del menor.

8. Categoría de publicidad:

- alimentación, bebidas y dulces

- belleza e higiene

- cultura y Educación

- deporte

- distribución y restauración

- electrónica

- entretenimiento

- famosos

- financiero

- juegos online

- moda

- músicos

- telecomunicaciones e internet

- transporte, viajes y turismo, y

- otros.

9. Marca expuesta en la publicidad. 


\section{Resultados}

El registro audiovisual que se toma como referencia para este estudio muestra que los niños pasan su tiempo de navegación mediante el teléfono móvil en YouTube, Instagram y en una gran variedad de aplicaciones de juegos ${ }^{1}$.

Los menores visitan YouTube casi una tercera parte del tiempo analizado, a través de la app (21\%) o desde el explorador (10\%). En cuanto a los juegos lo más destacable es la variedad de opciones (24 alternativas con un tiempo de uso de al menos 10 minutos). Aunque el acceso a juegos se realiza principalmente a través de apps (28\%), también es reseñable el acceso a juegos online desde el explorador (4\% del tiempo de navegación registrado). Los juegos que atraen más su atención son Candy Crush, Tetris y Clash Royale.

En lo que respecta a redes sociales, Instagram se posiciona como la segunda aplicación en la que pasan más tiempo de navegación (16\%). WhatsApp y Facebook (a la que se accede en mayor proporción desde el explorador que a través de la app) presentan un uso anecdótico. También conviene destacar el uso de TikTok (3\%) y el tiempo dedicado a búsquedas por el explorador del móvil (3\%).

Esta rutina de uso presenta diferencias en función de la propiedad del dispositivo. Si el móvil pertenece al menor, el $21 \%$ del tiempo de navegación transcurre en Instagram; en el caso de que el móvil sea del adulto se contabiliza un menor tiempo de consumo. Aunque YouTube es la aplicación con más tiempo de uso en los dos

\section{Los niños pasan su tiempo de navegación mediante el teléfono móvil en YouTube, Instagram y en una gran variedad de aplicaciones de juegos} segmentos, cuando se trata de móvil prestado los niños y niñas acceden principalmente a través del explorador, mientras que los que tienen un dispositivo propio lo hacen a través de la app. Los juegos están presentes en ambos casos, aunque a través de distintas opciones.

El sexo de los menores también marca algunas diferencias: YouTube es la plataforma que concentra más tiempo de uso tanto por niños como por niñas, pero de una forma más acusada en el caso de los varones (28\%). Las niñas tienden a acceder más a través del explorador. Instagram y los juegos presentan un porcentaje de uso similar entre niños y niñas, aunque hay divergencia en el tipo de juego consumido. El uso registrado de TikTok proviene fundamentalmente de dispositivos usados por niñas.

Tabla 1. Las cinco plataformas más visitadas categorizadas por propiedad del teléfono, sexo y edad

\begin{tabular}{|c|c|c|c|c|c|}
\hline Aplicaciones & $\begin{array}{c}\text { Tiempo de } \\
\text { navegación }\end{array}$ & $\begin{array}{c}\text { \% sobre } \\
\text { tiempo de } \\
\text { navegación }\end{array}$ & Aplicaciones & $\begin{array}{l}\text { Tiempo de } \\
\text { navegación }\end{array}$ & $\begin{array}{c}\text { \% sobre } \\
\text { tiempo de } \\
\text { navegación }\end{array}$ \\
\hline \multicolumn{3}{|c|}{ Móvil propiedad del menor } & \multicolumn{3}{|c|}{ Móvil propiedad de los padres } \\
\hline YouTube (app) & $6: 21: 34$ & 27 & YouTube (explorador) & $3: 29: 58$ & 20 \\
\hline Instagram (app) & 5:07:16 & 21 & YouTube (app) & $2: 35: 46$ & 15 \\
\hline Juego app (Candy Crush) & 1:16:07 & 5 & Instagram (app) & 1:44:56 & 10 \\
\hline Juego app (Clash Royale) & $0: 51: 19$ & 4 & Juego app (Tetris) & $1: 18: 46$ & 7 \\
\hline YouTube (explorador) & $0: 47: 19$ & 3 & TikTok & 0:50:58 & 5 \\
\hline \multicolumn{3}{|l|}{ Niño } & \multicolumn{3}{|l|}{ Niña } \\
\hline YouTube (app) & $3: 37: 07$ & 28 & YouTube (app) & $5: 20: 13$ & 18 \\
\hline Instagram (app) & $1: 50: 43$ & 15 & Instagram (app) & $5: 01: 29$ & 17 \\
\hline Juego app (Tetris) & $1: 23: 45$ & 11 & YouTube (explorador) & $3: 20: 26$ & 11 \\
\hline YouTube (explorador) & $0: 56: 51$ & 7 & Juego app (Candy Crush) & $1: 53: 54$ & 7 \\
\hline Juego app (Clash Royale) & $0: 51: 19$ & 7 & TikTok & $1: 28: 51$ & 5 \\
\hline \multicolumn{3}{|l|}{ 10-12 años } & \multicolumn{3}{|l|}{ 13-14 años } \\
\hline YouTube (app) & $5: 39: 23$ & 25 & Instagram (арр) & $4: 57: 56$ & 27 \\
\hline YouTube (explorador) & $2: 58: 00$ & 13 & YouTube (app) & $3: 17: 57$ & 18 \\
\hline Instagram (app) & $1: 54: 16$ & 8 & YouTube (explorador) & $1: 19: 17$ & 7 \\
\hline TikTok & $1: 23: 30$ & 6 & Juego app (Candy Crush) & $1: 16: 07$ & 7 \\
\hline Juego app (Tetris) & 0:41:55 & 3 & Juego app (Clash Royale) & $0: 51: 19$ & 5 \\
\hline
\end{tabular}

Si se segmenta el uso por edad, los menores entre 10-12 años dedican casi el 40\% de su tiempo de navegación a YouTube, un uso menos significativo entre el público de 13 a 14 años, que hace un uso mayor de Instagram. El consumo de TikTok proviene de teléfonos de niños y niñas más pequeños.

Tras describir el tipo de uso que la muestra realizó de sus dispositivos móviles, se pasa a analizar el impacto publicitario recibido. Es necesario aclarar que en este estudio se entiende por impacto publicitario y/o presencia publicitaria la permanencia en pantalla de cualquier mensaje de carácter comercial, es decir, se contabiliza el período de tiempo que 
la publicidad está expuesta en la pantalla del teléfono del sujeto. Así, se puede destacar que en el $23,4 \%$ del tiempo analizado se detectó presencia publicitaria en la pantalla grabada (14 minutos de publicidad por hora de navegación), contabilizando un total de 2.102 impresiones publicitarias, lo que equivale a una media de 50 anuncios por hora de uso del móvil. En los dispositivos que pertenecen al adulto se contabilizó más tiempo de publicidad que el dato medio (16 minutos de publicidad por hora de grabación), mientras que en los aparatos de uso exclusivo por parte del menor fue menor la exposición de mensajes comerciales (13 minutos). En cuanto al sexo, de forma general los niños fueron impactados durante más tiempo que las niñas, cuyo nivel de exposición fue más reducido.

Tabla 2. Tiempo de impacto publicitario con respecto al total de horas de navegación

\section{TIEMPO DE IMPACTO PUBLICITARIO}

9 horas con 45 minutos y 28 segundos

$23,4 \%$ del tiempo de navegación en el móvil, los menores fueron impactados por publicidad $=14$ minutos de publicidad $\mathrm{x}$ hora de navegación

2102 anuncios publicitarios

\begin{tabular}{|c|c|c|c|}
\hline $\begin{array}{c}\% \text { tiempo de navegación con } \\
\text { publicidad }\end{array}$ & $\begin{array}{l}\text { Minutos de publicidad / hora } \\
\text { de navegación }\end{array}$ & $\begin{array}{c}\text { \% tiempo de navegación con } \\
\text { publicidad }\end{array}$ & $\begin{array}{l}\text { Minutos de publicidad / hora } \\
\text { de navegación }\end{array}$ \\
\hline \multicolumn{2}{|c|}{ Móvil propiedad del menor } & \multicolumn{2}{|c|}{ Móvil propiedad de los padres } \\
\hline 22 & $13^{\prime}$ & 26 & $16^{\prime}$ \\
\hline \multicolumn{2}{|c|}{ Niño } & \multicolumn{2}{|c|}{ Niña } \\
\hline 26 & $16^{\prime}$ & 22 & $13^{\prime}$ \\
\hline \multicolumn{2}{|c|}{ 10-12 años } & \multicolumn{2}{|c|}{ 13-14 años } \\
\hline 24 & $14^{\prime}$ & 23 & $14^{\prime}$ \\
\hline
\end{tabular}

\subsection{Presencia publicitaria por aplicación}

YouTube (app y explorador) e Instagram representan el 35\% del total del tiempo catalogado como publicitario y un $50 \%$ del total de los anuncios detectados ${ }^{2}$. No hay que olvidar que se trata de las dos plataformas de más uso por parte de los niños y niñas que participaron en este estudio.

Si se analiza la densidad publicitaria en las apps más empleadas (tiempo de navegación en el que hay presencia de publicidad), YouTube alcanza un 10\% de exposición publicitaria a través de la app (equivalente a 6 minutos de publicidad por hora de navegación); si se accede a través del explorador, el tiempo de publicidad se incrementa al 15\%.

En lo que respecta a redes sociales, en Instagram hay mayor presencia de mensajes comerciales, llegando a estar en pantalla hasta un $20 \%$ del tiempo de uso (12 minutos por hora). Facebook, aunque no es una de las plataformas más populares entre los menores, también destaca por el nivel de presencia de publicidad, superior al de Instagram, con un $36 \%$ de tiempo de exhibición publicitaria (22 minutos por hora).

Con relación a las apps de juegos más usadas, se puede destacar que a excepción de Candy Crush, esta forma de entretenimiento se caracteriza por una "omnipresencia" de mensajes comerciales mientras se juega, pues entre el $40 \%$ y el $90 \%$ del tiempo se identificó publicidad. Hay casos en los que se registraron hasta 53 minutos de exhibición publicitaria por hora de navegación (Drive \& Park y Line Puzzle). A excepción de Candy Crush (que es la opción más popular), todos los demás títulos tienen un alto nivel de presión publicitaria.
Los canales principales de exposición a la publicidad son distintos según la propiedad del teléfono: si el móvil pertenece al menor son Instagram y YouTube y si el dispositivo es de sus padres, son los juegos

Los canales principales de exposición a la publicidad son distintos según la propiedad del teléfono:

- si el móvil pertenece al menor, el mayor periodo de consumo publicitario se concentra en Instagram y YouTube;

- si accede desde el dispositivo de un progenitor, la exposición a la publicidad viene de la mano de los juegos.

Si se diferencia por sexo, los móviles utilizados por niñas registran un volumen mayor de publicidad a través de Instagram, en comparación con los niños, que registran mayor impacto publicitario a través de las apps de juegos y de YouTube (app). 
La edad del menor también marca diferencias, pues en los móviles de usuarios más jóvenes (10-12 años) los datos muestran más tiempo de publicidad que la media en las plataformas de juegos (Aquapark.io y Drive \& Park); sin embargo, en los móviles de menores de más edad (13-14 años), se duplica el tiempo de exposición publicitaria en Instagram.

Tabla 3. Plataformas con más presencia publicitaria categorizadas por propiedad del teléfono, sexo y edad

\begin{tabular}{|c|c|c|c|c|c|c|c|c|c|}
\hline Aplicaciones & $\begin{array}{l}\text { Tiempo de } \\
\text { publicidad }\end{array}$ & $\begin{array}{l}\text { \% sobre } \\
\text { tiempo } \\
\text { publici- } \\
\text { dad }\end{array}$ & $\mathbf{N}$ & $\%$ & Aplicaciones & $\begin{array}{c}\text { Tiempo } \\
\text { publicidad }\end{array}$ & $\begin{array}{l}\text { \% sobre } \\
\text { tiempo } \\
\text { publici- } \\
\text { dad }\end{array}$ & $\mathbf{N}$ & $\%$ \\
\hline \multicolumn{3}{|c|}{ Móvil propiedad del menor } & & & \multicolumn{3}{|c|}{ Móvil propiedad de los padres } & & \\
\hline Instagram (app) & $1: 16: 24$ & 25 & 713 & 55 & Juego app (Tetris) & $1: 04: 30$ & 23 & 71 & 9 \\
\hline YouTube (app) & $0: 57: 50$ & 19 & 126 & 10 & YouTube (app) & $0: 40: 53$ & 15 & 115 & 14 \\
\hline Juego app (Stackball) & $0: 16: 38$ & 5 & 49 & 4 & Juego app (Aquapark.io) & $0: 21: 26$ & 8 & 53 & 7 \\
\hline $\begin{array}{l}\text { Juego app (Drive \& } \\
\text { Park) }\end{array}$ & $0: 16: 29$ & 5 & 40 & 3 & YouTube (explorador) & $0: 18: 20$ & 7 & 73 & 9 \\
\hline $\begin{array}{l}\text { Juego app (Super } \\
\text { Slimes) }\end{array}$ & 0:16:04 & 5 & 2 & 0 & Juego app (Bob's world) & 0:15:55 & 6 & 96 & 12 \\
\hline \multicolumn{3}{|l|}{ Niño } & & & \multicolumn{3}{|l|}{ Niña } & & \\
\hline Juego app (Tetris) & $1: 04: 31$ & 33 & 72 & 13 & Instagram (арр) & $1: 12: 47$ & 19 & 673 & 43 \\
\hline YouTube (app) & $0: 44: 50$ & 23 & 128 & 24 & YouTube (app) & $0: 53: 53$ & 1 & 113 & 7 \\
\hline $\begin{array}{l}\text { Juego app (Drive \& } \\
\text { Park) }\end{array}$ & $0: 16: 29$ & 8 & 40 & 7 & YouTube (explorador) & $0: 22: 22$ & 6 & 76 & 5 \\
\hline $\begin{array}{l}\text { Juego app (Pixel Shot } \\
\text { 3D) }\end{array}$ & $0: 10: 51$ & 5 & 27 & 5 & Juego app (Aquapark.io) & 0:19:14 & 5 & 45 & 3 \\
\hline $\begin{array}{l}\text { Juego app (Words of } \\
\text { Wonders) }\end{array}$ & 0:10:02 & 5 & 18 & 3 & Juego app (Stackball) & $0: 16: 38$ & 4 & 49 & 3 \\
\hline \multicolumn{3}{|l|}{ 10-12 años } & & & \multicolumn{3}{|l|}{ 13-14 años } & & \\
\hline YouTube (app) & 1:03:38 & 19 & 154 & 16 & Instagram (арр) & $1: 13: 51$ & 29 & 685 & 61 \\
\hline Juego app (Tetris) & $0: 31: 21$ & 9 & 41 & 4 & YouTube (app) & $0: 35: 05$ & 14 & 87 & 8 \\
\hline $\begin{array}{l}\text { Juego app (Aquapark. } \\
\text { io) }\end{array}$ & $0: 21: 20$ & 6 & 51 & 5 & Juego app (Tetris) & $0: 33: 10$ & 13 & 31 & 3 \\
\hline $\begin{array}{l}\text { Juego app (Drive \& } \\
\text { Park) }\end{array}$ & $0: 16: 29$ & 5 & 40 & 4 & Juego app (Stackball) & $0: 13: 44$ & 5 & 36 & 3 \\
\hline YouTube (explorador) & $0: 16: 17$ & 5 & 66 & 7 & Juego app (Pixel Shot 3D) & $0: 10: 51$ & 4 & 27 & 2 \\
\hline \multicolumn{10}{|c|}{$\begin{array}{l}\text { Aplicaciones = apps que empleó el menor en las grabaciones registradas. } \\
\text { Tiempo de publicidad = tiempo en el que se detectó presencia publicitaria en pantalla (hh:mm:ss) por app y perfil de menor. } \\
\% \text { sobre tiempo publicidad }=\% \text { del tiempo con presencia publicitaria en cada app sobre el total de tiempo de publicidad registrado para cada } \\
\text { perfil de menor. } \\
\mathrm{N}=\text { número total de anuncios contabilizados por app y perfil del menor. } \\
\%=\text { peso que cada app representa sobre el total de anuncios registrados para cada perfil de menor. }\end{array}$} \\
\hline
\end{tabular}

Ya se mencionó que los registros tomados desde dispositivos que pertenecen a los padres presentan un mayor impacto de publicidad, especialmente cuando se consumen juegos, donde el menor recibe un nivel de impacto casi permanente. También es reseñable cómo una cuarta parte del tiempo destinado a Instagram en niños y niñas con propiedad exclusiva del teléfono está presente la publicidad. Si se comparan los datos de exposición de publicidad en Instagram entre menores con móvil propio o ajeno, parece vislumbrarse que a más tiempo de uso, mayor proporción de presencia publicitaria en dicha red social.

Si se atiende al sexo, en sus rutinas los niños están mucho más tiempo expuestos a publicidad cuando juegan al Tetris, mientras que entre las niñas la mayor exposición publicitaria sucede en Instagram, aunque en menor proporción.

En cuanto a la edad, los más pequeños están sujetos a una exposición comercial casi permanente cuando juegan a través del móvil; en cambio, los de 13 a 14 años presentan unas rutinas de navegación menos saturadas de publicidad.

También resulta curioso cómo las apps de juegos que tienen una mayor saturación publicitaria son de uso frecuente entre los menores de menos edad, muy probablemente más tolerantes con la presencia de mensajes comerciales. Con todo, Tetris supondría una excepción pues a pesar de su alto porcentaje de contenido publicitario, es empleado indistintamente por todos los bloques de edad.

Los niños más pequeños están sujetos a una exposición comercial casi permanente cuando juegan a través del móvil 
Tabla 4. Impacto publicitario en las plataformas más empleadas por cada segmento de usuario analizado

\begin{tabular}{|c|c|c|c|c|c|}
\hline Aplicaciones & $\begin{array}{l}\% \text { de tiempo } \\
\text { de navegación } \\
\text { con publicidad }\end{array}$ & $\begin{array}{l}\text { Minutos de pu- } \\
\text { blicidad / hora } \\
\text { de navegación }\end{array}$ & Aplicaciones & $\begin{array}{l}\% \text { de tiempo } \\
\text { de navegación } \\
\text { con publicidad }\end{array}$ & $\begin{array}{l}\text { Minutos de pu- } \\
\text { blicidad / hora } \\
\text { de navegación }\end{array}$ \\
\hline \multicolumn{3}{|c|}{ Móvil propiedad del menor } & \multicolumn{3}{|c|}{ Móvil propiedad de los padres } \\
\hline YouTube (app) & 15 & 9 & YouTube (explorador) & 9 & 5 \\
\hline Instagram (app) & 25 & 15 & YouTube (app) & 26 & 16 \\
\hline Juego app (Candy Crush) & 8 & 5 & Instagram (app) & 5 & 3 \\
\hline Juego app (Clash Royale) & 6 & 4 & Juego app (Tetris) & 82 & 49 \\
\hline YouTube (explorador) & 13 & 8 & TikTok & 6 & 4 \\
\hline \multicolumn{3}{|l|}{ Niño } & \multicolumn{3}{|l|}{ Niña } \\
\hline YouTube (app) & 21 & 13 & YouTube (app) & 17 & 10 \\
\hline Instagram (app) & 8 & 5 & Instagram (app) & 24 & 14 \\
\hline Juego app (Tetris) & 77 & 46 & YouTube (explorador) & 1 & 7 \\
\hline YouTube (explorador) & 5 & 3 & Juego app (Candy Crush) & 6 & 4 \\
\hline Juego app (Clash Royale) & 6 & 4 & TikTok & 3 & 2 \\
\hline \multicolumn{3}{|l|}{ 10-12 años } & \multicolumn{3}{|l|}{ 13-14 años } \\
\hline YouTube (app) & 19 & 11 & Instagram (app) & 25 & 15 \\
\hline YouTube (explorador) & 9 & 5 & YouTube (app) & 18 & 11 \\
\hline Instagram (app) & 7 & 4 & YouTube (explorador) & 10 & 6 \\
\hline TikTok & 4 & 2 & Juego app (Candy Crush) & 8 & 5 \\
\hline Juego app (Tetris) & 75 & 45 & Juego app (Clash Royale) & 6 & 4 \\
\hline
\end{tabular}

Tabla 5. Tipo de publicidad recibida según propiedad del teléfono, sexo y edad

\begin{tabular}{|c|c|c|c|c|c|c|c|c|c|}
\hline Categoría & $\begin{array}{l}\text { Tiempo } \\
\text { de publi- } \\
\text { cidad }\end{array}$ & $\begin{array}{c}\text { \% sobre } \\
\text { tiempo } \\
\text { publici- } \\
\text { dad }\end{array}$ & $\mathbf{N}$ & $\%$ & Categoría & $\begin{array}{l}\text { Tiempo } \\
\text { de publi- } \\
\text { cidad }\end{array}$ & $\begin{array}{l}\% \text { sobre } \\
\text { tiempo } \\
\text { publici- } \\
\text { dad }\end{array}$ & $\mathbf{N}$ & $\%$ \\
\hline \multicolumn{3}{|c|}{ Móvil propiedad del menor } & & & \multicolumn{3}{|c|}{ Móvil propiedad de los padres } & & \\
\hline Juegos online & 1:16:11 & 25 & 160 & 12 & Distribución/restauración & $1: 02: 47$ & 23 & 174 & 22 \\
\hline Distribución/restauración & $0: 45: 14$ & 15 & 96 & 7 & Juegos online & $0: 59: 37$ & 22 & 169 & 21 \\
\hline Entretenimiento & $0: 22: 45$ & 7 & 107 & 8 & Moda & $0: 29: 16$ & 11 & 43 & 5 \\
\hline Transporte/viajes/turismo & $0: 21: 15$ & 7 & 117 & 9 & Transporte/viajes/turismo & $0: 22: 35$ & 8 & 74 & 9 \\
\hline $\begin{array}{l}\text { Alimentación/bebidas/ } \\
\text { dulces }\end{array}$ & $0: 18: 39$ & 6 & 110 & 8 & Entretenimiento & $0: 23: 22$ & 8 & 63 & 8 \\
\hline \multicolumn{3}{|l|}{ Niños } & & & \multicolumn{3}{|l|}{ Niñas } & & \\
\hline Juegos online & 0:40:55 & 21 & 119 & 22 & Juegos online & $1: 34: 53$ & 25 & 210 & 13 \\
\hline Distribución/restauración & 0:43:47 & 22 & 83 & 15 & Distribución/restauración & 1:04:14 & 17 & 187 & 12 \\
\hline Moda & $0: 21: 40$ & 11 & 26 & 5 & Transporte/viajes/turismo & $0: 25: 37$ & 7 & 122 & 8 \\
\hline Entretenimiento & $0: 20: 40$ & 10 & 59 & 11 & Entretenimiento & $0: 25: 27$ & 7 & 111 & 7 \\
\hline Transporte/viajes/turismo & 0:18:13 & 9 & 69 & 13 & Moda & 0:23:18 & 6 & 218 & 14 \\
\hline \multicolumn{3}{|l|}{ 10-12 años } & & & \multicolumn{3}{|l|}{ 13-14 años } & & \\
\hline Juegos online & 1:35:13 & 29 & 230 & 24 & Distribución/restauración & 0:49:20 & 19 & 105 & 9 \\
\hline Distribución/restauración & 0:58:41 & 18 & 165 & 17 & Juegos online & $0: 40: 35$ & 16 & 99 & 9 \\
\hline Transporte/viajes/turismo & $0: 28: 24$ & 9 & 102 & 11 & Moda & $0: 28: 34$ & 11 & 198 & 18 \\
\hline Entretenimiento & $0: 27: 00$ & 8 & 69 & 7 & Entretenimiento & 0:19:07 & 8 & 101 & 9 \\
\hline Moda & $0: 16: 24$ & 5 & 46 & 5 & Belleza/higiene & $0: 17: 24$ & 7 & 98 & 9 \\
\hline \multicolumn{10}{|c|}{$\begin{array}{l}\text { Categoría = sector al que pertenece el producto/servicio anunciado en las publicidades registradas. } \\
\text { Tiempo de publicidad = tiempo en el que se detectó presencia publicitaria en pantalla (hh:mm:ss) por categoría de producto y perfil de menor. } \\
\% \text { sobre tiempo publicidad= \% del tiempo de cada categoría de producto/servicio sobre el total de tiempo de publicidad registrado para cada } \\
\text { perfil de menor. } \\
\mathrm{N}=\text { número total de anuncios contabilizados por categoría de producto/servicio y perfil del menor. } \\
\%=\text { peso que cada categoría de producto/servicio representa sobre el total de anuncios registrados para cada perfil de menor. }\end{array}$} \\
\hline
\end{tabular}




\subsection{Presencia publicitaria por categoría de producto anunciado}

Si se clasifican los anuncios publicitarios por categoría de producto, lo más destacable es la diversidad de sectores de los que reciben publicidad los menores: 17 categorías más una de "Otros" que contempla una amplia variedad de sectores que por sí solos eran anecdóticos, pero que sumándolos concentran un volumen importante de mensajes comerciales (inmobiliarias, sellos discográficos, aplicaciones de georreferenciación, aplicaciones de traducción, ópticas, clínicas...).

La publicidad sobre juegos online, distribución y restauración, entretenimiento, moda y transporte y viajes representan más de la mitad del tiempo de exposición comercial y del volumen de anuncios contabilizados.

La propiedad del teléfono, el sexo y la edad marcan diferencias en cuanto al tipo de publicidad recibida: en los dispositivos de uso propio, los mensajes sobre alimentación, bebidas y dulces están más presentes que en los móviles prestados, en los que adquieren más peso los anuncios sobre distribución y restauración y sobre juegos online. Aunque el sexo no supone grandes diferencias, sí que se puede diferenciar una mayor tendencia a la publicidad de moda en los móviles empleados por niñas, así como de juegos online en los gestionados por niños. Si se segmenta por edad, los sectores de moda y de belleza e higiene se hacen más presentes en las rutinas de navegación de los usuarios entre 13 y 14 años.

\section{La interacción que mantiene el menor con la publicidad analizada es preferen- temente visual, no obstante, en los dis- positivos que son propiedad del menor se percibe mayor interacción}

\subsection{Tipo de relación mantenida con la publicidad}

La interacción que mantiene el menor con la publicidad analizada es preferentemente visual, esto es, el anuncio aparece visible en la pantalla sin mayor vínculo que un contacto ocular. En un $9 \%$ de los casos analizados el nivel de interacción con la publicidad fue mayor: se le presta mayor atención observándola por un tiempo prolongado o cerrándola. La interacción a través del click sólo sucede en un $2 \%$ de los casos.

En los dispositivos que son propiedad del menor se percibe una mayor interacción con la publicidad. De igual manera, los móviles usados por niñas tienden a presentar un mayor nivel de interacción con la publicidad que los utilizados por varones.

\section{Discusión}

Se pone de manifiesto que la densidad publicitaria detectada a través del teléfono móvil a la que se exponen los menores merece una atención especial. Medir el impacto publicitario que reciben en este dispositivo es una tarea compleja que se ha afrontado con una metodología que, pese a que impuso una serie de limitaciones, permite establecer unas primeras bases y líneas de debate sobre el tema.

Los manuales sobre publicidad subrayan la saturación publicitaria como una de las principales razones por las que los medios convencionales perdieron su liderazgo a favor de los no convencionales, más liberados de mensajes comerciales y con una estrategia de llegada al consumidor más personal y directa. No obstante, esta investigación arroja que en términos generales la publicidad estuvo presente en el 23,4\% del tiempo total de navegación, lo que significa que por cada hora de uso se registraron alrededor de 14 minutos de publicidad. Si se compara esta densidad publicitaria con la de la televisión, que es uno de los principales medios en cuanto inversión publicitaria (Infoadex, 2019; AAM, 2019), se puede comprobar que comparten unas estadísticas similares: así, en España el artículo 14 de la Ley General de la Comunicación Audiovisual (España, 2010) estipula un máximo de 12 minutos de mensajes publicitarios por hora.

En el caso de Chile, los últimos anuarios de publicidad en televisión abierta disponibles y publicados por el Consejo Nacional de Televisión (2010; 2011), reflejaron una media de entre 8 y 10 minutos de mensajes comerciales por hora de emisión. Puede deducirse que al menos en el contexto estudiado, los menores visualizan un volumen de mensajes comerciales a través del móvil superior a lo que se exhibe en televisión, medio que se caracteriza por su saturación publicitaria. La diferencia radica en que la presión publicitaria ejercida a través del móvil pasa más desapercibida por el consumo individual del dispositivo. Por ello la mediación parental se hace más compleja, aunque más necesaria si cabe.

YouTube, Instagram y las apps de videojuegos se convierten en las principales plataformas donde el menor es impactado por publicidad. De las tres, los juegos presentaron una densidad publicitaria mucho mayor que la media establecida. Se comprobó que en algunos juegos utilizados por la muestra, la exhibición de mensajes comerciales fue continua durante casi todo el tiempo de permanencia en la app. Se trata de un dato relevante por el nivel que alcanzan los juegos en las rutinas de uso del teléfono móvil de los menores, especialmente de los más pequeños, al tratarse de una de sus principales formas de entretenimiento.

La propiedad del dispositivo resultó significativa en el análisis de la relación entre el menor y la publicidad recibida. Los menores dueños de sus móviles reflejaron un uso más diverso sobre el aparato y sobre la navegación

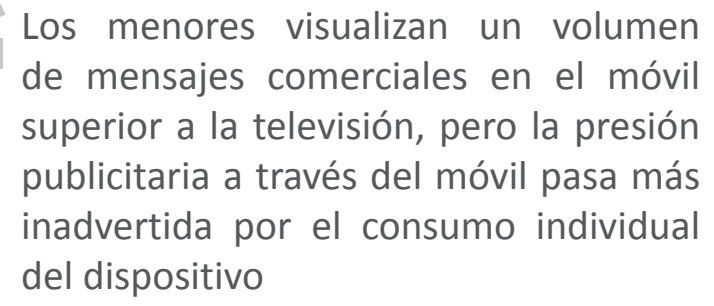
superior a la televisión, pero la presión publicitaria a través del móvil pasa más inadvertida por el consumo individual del dispositivo 
a través del móvil, mientras que los que hicieron uso de aparatos de sus padres mostraron un uso más restringido, limitándose a la navegación en YouTube y uso de juegos. Que el menor utilice un dispositivo de sus padres hace que su navegación sea más acotada, pero recibe más publicidad que quienes tienen móvil propio, especialmente a través de los juegos y sobre marcas y productos alejados de sus intereses. Se abre por tanto una línea de debate que podría ser interesante abordar en futuras investigaciones sobre la idoneidad de los mensajes comerciales que los más pequeños visualizan cuando acceden a través de los aparatos móviles de sus progenitores, ya que a través de estas pantallas se reciben anuncios personalizados acordes a las rutinas de navegación y al perfil de usuario registrado, en este caso un adulto, pero que acaban impactando en niños.

Relacionado con lo anterior, se detectó que el menor es alcanzado por un amplio abanico de productos y marcas pertenecientes a categorías a priori poco relacionadas con los intereses de un usuario entre 10 y 14 años. Si bien es cierto que en el $25 \%$ del tiempo publicitario detectado se anunciaron juegos online afines a las rutinas de uso de los menores, otras categorías como distribución y restauración y transporte, viajes y turismo también representan un peso importante en la oferta publicitaria, especialmente a través del móvil del adulto, en el que otros sectores como automoción o financiero también son más frecuentes. Cuando el dispositivo era propiedad del menor se mostraron anuncios más acordes a sus inquietudes (belleza, alimentación, bebidas y dulces, músicos, youtubers), no obstante, las categorías más predominantes siguieron siendo juegos online y distribución y restauración. Este hallazgo da pie a reflexionar que a pesar de que la rutina de navegación del menor sea acorde a su edad y que cuente con la aprobación de sus padres, el menor puede estar siendo impactado simultáneamente por mensajes comerciales de productos y marcas de los que no es público objetivo.

Como se pudo comprobar en los resultados, la relación mantenida con la publicidad es principalmente visual, esto es, los mensajes comerciales aparecen en pantalla sin mayor interacción por parte del menor. Aun así, el 11\% de la muestra prestó más nivel de atención al mensaje comercial, aunque fuese para cerrarlo. Aunque no sea objetivo de esta investigación, es en este punto donde entra a discusión la efectividad de este tipo de formatos publicitarios móviles, que como ya se ha apuntado en los estudios citados, es poco recordada, especialmente los anuncios en apps de videojuegos.

Los menores son conscientes de la densidad publicitaria a la que se enfrentan cuando acceden a los dispositivos móviles y son capaces de identificar las formas que adquieren los mensajes comerciales en el contexto digital. Sin embargo, queda pendiente comprobar si también reconocen y se cuestionan el carácter persuasivo del contenido, sobre todo en los casos en los que la publicidad no emplea las formas convencionales a las que los niños están acostumbrados. Se abre una línea de investigación en la que seguir trabajando, sobre todo centrada en la publicidad más encubierta (emplazamiento o recomendación de productos por parte de influencers) y la capacidad de los menores para reconocer y cuestionarse la intencionalidad persuasiva de estos contenidos, una fase ya contemplada en el proyecto de investigación (Fondecyt 11170336) en el que se enmarca este estudio.

\section{Conclusiones}

Si bien es cierto que el método empleado puede haber coartado a la muestra de emplear otros canales más personales (por ejemplo, WhatsApp), los resultados evidencian un uso preferentemente encaminado al entretenimiento y a la consulta de Instagram, red social muy visual. Es a través de estas plataformas, YouTube, apps de juegos e Instagram, por las que el menor recibe más publicidad durante sus rutinas de navegación que se traduce en una media de 14 minutos por hora, en proporciones ligeramente superiores a la densidad publicitaria de otros medios convencionales como la televisión, caracterizado por su nivel de saturación publicitaria. Mención aparte merece la presencia de mensajes comerciales en las apps de juegos en los que la densidad publicitaria puede llegar al casi $90 \%$ del tiempo de uso de la aplicación, lo que se traduce en una exhibición constante de productos y marcas mientras el niño se entretiene jugando.

También se comprobó que la relación mantenida con este tipo de publicidad es principalmente visual, no obstante, los menores con móvil propio y las niñas tienden a mantener un mayor nivel de interacción con los mensajes comerciales. En relación a las preguntas de investigación planteadas, se comprobó que efectivamente la propiedad del teléfono marca diferencias en la exposición publicitaria, no solo en la relación establecida con la publicidad, sino que también influye en el volumen de publicidad recibida -los que acceden desde el dispositivo de sus padres son impactados por más tiempo por publicidad que los que tienen móvil propio-y en el tipo de anunciantes, pertenecientes en una importante proporción a categorías de productos y marcas a priori poco relacionadas con los intereses de los más pequeños.

Esta investigación, que busca aportar un diagnóstico inicial sobre el impacto publicitario que los menores reciben a través de las pantallas de su móvil, arroja información que resulta útil para reconsiderar las bases de una alfabetización publicitaria que tenga en cuenta las particularidades de estas nuevas pantallas caracterizadas por un uso individual. La dificultad de la mediación parental en estas nuevas situaciones de consumo personal hace mucho más necesaria la labor de los educomunicadores, que debería también adaptarse tanto a los formatos

En las apps de juegos la densidad publicitaria puede llegar a casi el $90 \%$ del tiempo de uso de la aplicación, lo que se traduce en una exhibición constante de productos y marcas mientras el niño se entretiene jugando 
como a las situaciones de consumo que se generan. La presión publicitaria que enfrentan los menores en el uso que hacen de internet debería interpelar a educadores, gobernantes y empresas. Este artículo proporciona evidencias científicas sobre las que sustentar posibles líneas de acción y políticas.

\section{Notas}

1. Dada la variedad de juegos visitados por los niños, se contabilizaron los juegos en los que se registraban al menos 10 minutos de uso.

2. Número de anuncios se traduce como las impresiones publicitarias visibles en la pantalla del móvil.

\section{Referencias}

AAM (2019). Inversión publicitaria en medios. Asociación de Agencias de Medios.

https://aam.cl/wp-content/uploads/2019/12/Informe-Inversi\%C3\%B3n-en-Medios_Octubre-2019_AAM_v1.pdf

An, Soontae; Jin, Hyun-Seung; Park, Eun-Hae (2014). “Children's advertising literacy for advergames: Perception of the game as advertising". Journal of advertising, v. 43, n. 1, pp. 63-72.

https://doi.org/10.1080/00913367.2013.795123

An, Soontae; Kang, Hannah (2014). "Advertising or games? Advergames on the internet gaming sites targeting children". International journal of advertising, v. 33, n. 3, pp. 509-532.

https://doi.org/10.2501/IJA-33-3-509-532

Bakardjieva, Maria (2005). Internet society: The Internet in everyday life. London: SAGE. ISBN: 9780761943396 https://doi.org/10.4135/9781446215616

Cabello, Patricio; Claro, Magdalena (2017). Presentación de datos preliminares. Encuesta kids online Chile. Santiago: Kids Online Chile/ PUCV.

Cabello, Patricio; Lazcano, Daniela; Claro, Magdalena; Dodel, Matías; Aguirre, Florencia; Senne, Fabio; Martínez, Ana-Laura; Osorio-Tamayo, Liliana; Moreno-Carmona, Norman; Pérez, Rolando; León, Laura (2018). “Niñez, adolescencia y tecnologías digitales en América Latina”. En: Jiménez, Estefanía; Garmendia, Maialen; Casado, Miguel-Ángel (eds.). Entre selfies y whatsapps: Oportunidades y riesgos para la infancia y la adolescencia conectada. Barcelona: Gedisa, pp. 353-372. ISBN: 9788416919871

https://www.researchgate.net/publication/326261964_Ninez_adolescencia_y_tecnologias_digitales_en_America_Latina

Chen, Ying; Zhu, Sencun; Xu, Heng; Zhou, Yilu (2013). “Children's exposure to mobile in-app advertising: An analysis of content appropriateness". In: SocialCom, 2013 International conference on social computing, pp. 196-203. ISBN: 9780 769551371

https://doi.org/10.1109/SocialCom.2013.36

Consejo Anual de Televisión (2010). Anuario de publicidad TV Abierta. Consejo Anual de Televisión. http://prontus.cntv.cl/cntv/site/artic/20111024/asocfile/20111024181902/anuario_de_publicidad_2010.pdf

Consejo Anual de Televisión (2011). Anuario de publicidad TV Abierta. Consejo Anual de Televisión. http://prontus.cntv.cl/cntv/site/artic/20121205/asocfile/20121205163515/anuario_de_publicidad_2011.pdf

Culp, Jennifer; Bell, Robert-Alan; Cassady, Diana (2010). “Characteristics of food industry web sites and 'advergames' targeting children". Journal of nutrition education and behavior, v. 42, n. 3, pp. 197-201.

https://doi.org/10.1016/j.jneb.2009.07.008

De-Frutos-Torres, Belinda; Pretel-Jiménez, Marilé; Sánchez-Valle, María (2014). “La interacción de los jóvenes con las marcas en las redes sociales: hacia la presencia consentida y deseada". adComunica, v. 7, pp. 69-86.

http://repositori.uji.es/xmlui/handle/10234/97461

De-Jans, Steffi; Van-de-Sompel, Dieneke; Hudders, Liselot; Cauberghe, Veroline (2017). “Advertising targeting young children: an overview of 10 years of research (2006-2016)". International journal of advertising, v. 38 , n. 2.

https://doi.org/10.1080/02650487.2017.1411056

Emarketer (2016). Key digital trends for 2016. Emarketer.

https://www.emarketer.com/Webinar/Key-Digital-Trends-2016/4000125

España (2010). “Ley 7/2010, de 31 de marzo, general de la comunicación audiovisual”. BOE, n. 79, 1 abril. https://www.boe.es/buscar/pdf/2010/BOE-A-2010-5292-consolidado.pdf

Feijoo-Fernández, Beatriz; García-González, Aurora (2019). “Actitud del menor ante la publicidad que recibe a través de los dispositivos móviles". adComunica, v. 18, pp. 199-218.

http://repositori.uji.es/xmlui/handle/10234/184057 
Harris, Jennifer L.; Speers, Sarah E.; Schwartz, Marlene B.; Brownell, Kelly D. (2012). “US food company branded advergames on the internet: Children's exposure and effects on snack consumption". Journal of children and media, v. 6, n. 1, pp. 51-68.

https://doi.org/10.1080/17482798.2011.633405

Hine, Christine (2004). Etnografía virtual. Barcelona: Editorial UOC. ISBN: 8497880196

https://seminariosocioantropologia.files.wordpress.com/2014/03/hine-christine-etnografia-virtual-uoc.pdf

Hudders, Liselot; De-Pauw, Pieter; Cauberghe, Veroline; Panic, Katarina; Zarouali, Brahim; Rozendaal, Esther (2017). "Shedding new light on how advertising literacy can affect children's processing of embedded advertising formats: a future research agenda". Journal of advertising, v. 46, n. 2, pp. 333-349.

https://doi.org/10.1080/00913367.2016.1269303

IMS; Comscore (2017). IMS mobile in Latam study. 2nd Edition. IMS\&Comscore.

https://www.imscorporate.com/news/Estudios-comScore/IMS-Mobile-Study-Septiembre2016.pdf

Infoadex (2019). Resumen. Estudio Infoadex de la inversión publicitaria en España 2018. Infoadex.

https://www.infoadex.es/home/wp-content/uploads/2018/02/Estudio-InfoAdex-2018.pdf

Kantar Millward Brown (2017). AdReaction: Engaging Gen X, Y and Z. Kantar.

https://iabeurope.eu/wp-content/uploads/2019/08/AdReaction-Gen-X-Y-and-Z_Global-Report_FINAL_Jan-10-2017.pdf

Kargo Research; Tobii (2019). Viewability vs. memorability. Your ads are seen, but are they remembered? Kargo Research \& Tobii.

http://bit.ly/2SVOLUC

Koslow, Scott (2000). "Can the truth hurt? How honest and persuasive advertising can unintentionally lead to increased consumer skepticism". Journal of consumer affairs, v. 34, n. 2, pp. 245-267.

https://doi.org/10.1111/j.1745-6606.2000.tb00093.x

Leung, Linda (2005). Virtual ethnicity: Race, resistance and the World Wide Web. London: Routledge. ISBN: 9781315234 991

https://doi.org/10.4324/9781315234991

Livingstone, Sonia (2003). "Children's use of the internet: Reflections on the emerging research agenda". New media and society, v. 5, n. 2, pp. 147-166.

https://doi.org/10.1177/1461444803005002001

Mascheroni, Giovanna; Ólafsson, Kjartan (2014). Net children go mobile: risks and opportunities. Milán: Educatt. ISBN: 9788867802883

http://netchildrengomobile.eu/ncgm/wp-content/uploads/2013/07/DEF_NCGM_SecondEdition_Report.pdf

Meyer, Marisa; Adkins, Victoria; Yuan, Nalingna; Weeks, Heidi M.; Chang, Yung-Ju; Radesky, Jenny (2019). “Advertising in young children's apps. A content analysis". Journal of developmental \& behavioral pediatrics, v. 40, n. 1, pp. 32-39. https://doi.org/10.1097/DBP.0000000000000622

Miller, Daniel; Slater, Don (2000). The internet: An ethnographic approach. Oxford: Berg. ISBN: 9781859733899

Moore, Elizabeth S. (2004). "Children and the changing world or advertising". Journal of business ethics, v. 52, n. 2, pp. 161-167.

https://doi.org/10.1023/B:BUSI.0000035907.66617.f5

Oates, Caroline; Newman, Nicki; Tziortzi, Athanasia (2014). "Parents' beliefs about, and attitudes towards, marketing to children". In: Blades, Mark; Oates, Caroline, Blumberg, Fran; Gunter, Barrie (eds.). Advertising to children: New directions, new media. New York: Springer, pp. 115- 136. ISBN: 9781137313256

https://doi.org/10.1057/9781137313256

OCDE (2011). Protection of children online. Organización para la Cooperación y el Desarrollo Económicos, OCDE. https://www.oecd-ilibrary.org/science-and-technology/the-protection-of-children-online_5kgcjf71p/28en;jsessionid=rFhlfSm-ar87dJVgCx6dCTeR.ip-10-240-5-180

Ofcom (2016). Communication market report 2016. Ofcom.

https://www.ofcom.org.uk/_data/assets/pdf_file/0024/26826/cmr_uk_2016.pdf

Pew Research Center (2018). Global attitudes survey. Pew Research Center.

https://pewrsr.ch/37hvQzv

Rozendaal, Esther; Slot, Noortje; Van-Reijmersdal, Eva A.; Buijzen, Moniek (2013). “Children's responses to advertising in social games". Journal of advertising, v. 42, n. 2-3, pp. 142-154.

https://doi.org/10.1080/00913367.2013.774588 
Sádaba-Chalezquer, Charo (2018). “Niños como consumidores digitales”. En: Jiménez, Estefanía; Garmendia, Maialen; Casado, Miguel-Ángel (eds.). Entre selfies y whatsapps: Oportunidades y riesgos para la infancia y la adolescencia conectada. Barcelona: Gedisa, pp. 289-301. ISBN: 9788416919871

Subtel (2017). IX Encuesta de acceso y usos de internet. Subtel.

https://www.subtel.gob.cl/wp-content/uploads/2018/07/Informe_Final_IX_Encuesta_Acceso_y_Usos_Internet_2017.pdf

Subtel (2019). Estadísticas sectoriales - Primer semestre 2019. Subtel.

https://www.subtel.gob.cl/usuarios-de-internet-movil-consumen-10-gb-en-promedio-al-mes-y-numero-de-telefonosmoviles-sube-a-26-millones

Terlutter, Ralf; Capella, Michael L. (2013). "The gamification of advertising: Analysis and research directions of in-game advertising, advergames, and advertising in social network games". Journal of advertising, v. 42, n. 2-3, pp. 95-112. https://doi.org/10.1080/00913367.2013.774610

Van-Reijmersdal, Eva A.; Rozendaal, Esther; Smink, Nadia; Van-Noort, Guda; Buijzen, Moniek (2017). “Processes and effects of targeted online advertising among children". International journal of advertising, v. 36, n. 3, pp. 396-414. https://doi.org/10.1080/02650487.2016.1196904

Vanwesenbeeck, Ini; Walrave, Michel; Ponnet, Koen (2017). "Children and advergames: the role of product involvement, prior brand attitude, persuasion knowledge and game attitude in purchase intentions and changing attitudes". International journal of advertising, v. 36, n. 4, pp. 520-541.

https://doi.org/10.1080/02650487.2016.1176637

Waiguny, Martin K. J.; Nelson, Michelle R.; Terlutter, Ralf (2012). “Entertainment matters! The relationship between challenge and persuasiveness of an advergame for children". Journal of marketing communications, v. 18, n. 1, pp. 69-89. https://doi.org/10.1080/13527266.2011.620766

YouGov (2017). Teen advertising. YouGov.

https://d25d2506sfb94s.cloudfront.net/cumulus_uploads/document/Ihojefz2cm/YouGov\%20NY\%20(Teen\%20 advertising)\%20103\%2005.25.2017.pdf

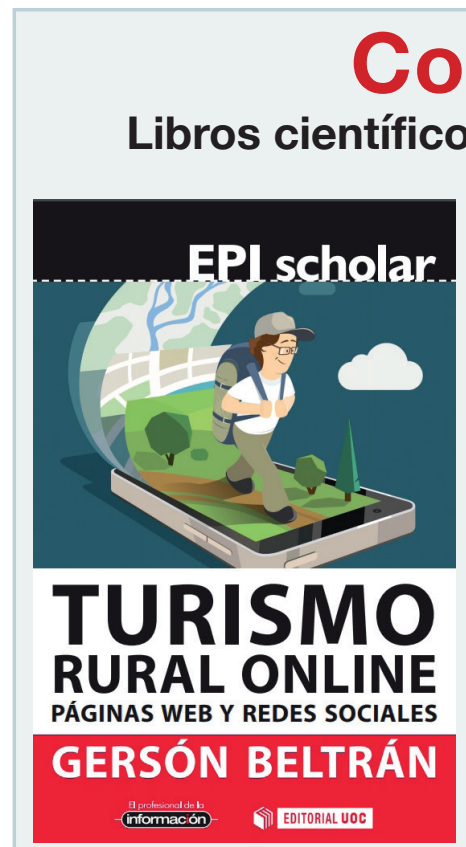

\section{Turismo rural online. Páginas web y redes sociales de Gersón Beltrán}

Los turistas están conectados en todo momento y se mueven en un entorno social, local y móvil: buscan información en internet antes de ir a su destino y realizan las reservas por anticipado, utilizan el móvil durante su experiencia turística para comunicarse y, tanto durante como tras el viaje, comparten sus opiniones en medios sociales.

Las empresas y destinos turísticos deben adaptarse a esta realidad, en la que lo físico es inseparable de lo online, conformando un entorno híbrido. Paralelamente, los espacios rurales sufren numerosos problemas de desarrollo y despoblación, y el turismo rural constituye una actividad que puede ayudar a revertir esta situación. Las nuevas tecnologías se configuran como herramientas que unen a los turistas conectados con estos espacios.

Este libro ofrece una visión general de la situación del turismo rural online, así como las claves para que las empresas y destinos turísticos logren conectar con los turistas y éstos disfruten de las experiencias únicas que les ofrece el turismo rural.

Beltrán, Gersón (2019). Turismo rural online. Páginas web y redes sociales. Barcelona: El profesional de la información, Editorial UOC, colección EPI Scholar n. 11, 170 pp. ISBN: 9788491803881

\section{Información}

http://www.elprofesionaldelainformacion.com/librosEPIScholar.html 